\title{
O uso de nanotecnologia em fármacos no Brasil
}

\author{
The use of nanotechnology in pharmaceuticals in Brazil \\ El uso de la nanotecnología en productos farmacéuticos en Brasil
}

\section{Resumo}

Com o avanço tecnológico no mundo todo ao longo dos anos, o uso de novas tecnologias que contribuíssem positivamente na qualidade de vida continua sendo alvo de muito investimento e estudos. A nanotecnologia ganha destaque neste contexto, pois, seu uso na busca pela melhoria na saúde vem trazendo bons resultados e seu emprego em fármacos traz grandes contribuições como a proteção ao organismo da degradação do fármaco, aumento da solubilidade e biodisponibilidade, diminuição de efeitos tóxicos e possíveis reações adversas e um maior controle e especificação na liberação dos fármacos. O Brasil é o país que possuí a maior e melhor infraestrutura da América Latina relacionados aos estudos da nanotecnologia com aplicação na área da saúde, porém no que se diz respeito sobre a produção o país se encontra em um nível intermediário. A pesquisa desenvolvida tem como principal objetivo descrever a situação do uso da nanotecnologia em fármacos no Brasil nos últimos 10 anos, as vantagens e desvantagens citadas entre a literatura cientifica e a importância e contribuição de mecanismos como esse para fins de diagnóstico e tratamento de doenças. Foi realizada uma revisão da literatura, sendo analisados estudos que abordassem a temática. Após toda a pesquisa e coleta de dados observou-se que a nanotecnologia e sua utilização em fármacos trazem muitos benefícios e auxiliam no tratamento de diversas doenças, porém seu efeito toxico ainda é muito pouco difundido pela falta de testes em fase clínica e pré-clínica e o Brasil possuí estrutura e investimento, mas falta incentivo e profissionais capacitados para realização de mais estudos e consequentes produções.

Palavras-chave: Nanotecnologia; Medicamentos e Tecnologia; Nanoestruturas; Nanotecnologia e fármacos.

\begin{abstract}
With technological advances worldwide over the years, the use of new technologies that contribute positively to quality of life continues to be the target of much investment and studies. Nanotechnology gains prominence in this context because its use in the search for health improvement has brought good results and its use in drugs brings great contributions such as the protection to the body of drug degradation, increased solubility and bioavailability, decreased toxic effects and possible adverse reactions and a greater control and specification in the release of drugs. Brazil is the country with the largest and best infrastructure in Latin America related to nanotechnology studies with application in the health area, but with regard to production the country is at an intermediate level. The main objective of the research developed is to describe the situation of the use of nanotechnology in drugs in Brazil in the last 10 years, the advantages and disadvantages mentioned among the scientific literature and the importance and contribution of mechanisms such as this for the purpose of diagnosis and treatment of diseases. A literature review was performed, analyzing studies addressing the theme. After all the research and data collection, it was observed that nanotechnology and its use in drugs bring many benefits and help in the treatment of various diseases, but its toxic effect is still very little widespread due to the lack of clinical and pre-clinical tests and Brazil has structure and investment, but lacks incentive and professionals trained to carry out more studies and consequent productions.
\end{abstract}

Keywords: Nanotechnology; Medicines and Technology; Nanostructures; Nanotechnology and pharmaceuticals.

\section{Resumen}

Con los avances tecnológicos en todo el mundo a lo largo de los años, el uso de nuevas tecnologías que contribuyen positivamente a la calidad de vida sigue siendo el objetivo de muchas inversiones y estudios. La nanotecnología gana 
protagonismo en este contexto porque su uso en la búsqueda de la mejora de la salud ha traído buenos resultados y su uso en fármacos aporta grandes aportes como la protección al organismo de la degradación de fármacos, aumento de la solubilidad y biodisponibilidad, disminución de efectos tóxicos y posibles reacciones adversas y un mayor control y especificación en la liberación de fármacos. Brasil es el país con la mayor y mejor infraestructura de América Latina relacionada con estudios de nanotecnología con aplicación en el área de la salud, pero con respecto a la producción el país se encuentra en un nivel intermedio. El objetivo principal de la investigación desarrollada es describir la situación del uso de la nanotecnología en medicamentos en Brasil en los últimos 10 años, las ventajas y desventajas mencionadas entre la literatura científica y la importancia y contribución de mecanismos como este para el diagnóstico y tratamiento de enfermedades. Se realizó una revisión de la literatura, analizando los estudios que abordan el tema. Después de toda la investigación y la recopilación de datos, se observó que la nanotecnología y su uso en medicamentos traen muchos beneficios y ayudan en el tratamiento de diversas enfermedades, pero su efecto tóxico aún está muy poco extendido debido a la falta de pruebas clínicas y preclínicas y Brasil tiene estructura e inversión, pero carece de incentivos y profesionales capacitados para realizar más estudios y producciones consecuentes.

Palabras clave: Nanotecnología; Medicamentos y Tecnología; Nanoestructuras; Nanotecnología y productos farmacéuticos.

\section{Introdução}

A nanotecnologia é uma área científica que surgiu a partir da ideia de criar uma tecnologia em escala nanométrica, que fosse útil em diferentes esferas, a fim de gerar melhoria na qualidade de vida. Pinto (2020) refere-se ao trabalho de Ferreir \& Rangel (2009) onde afirmam que a ideia de nanotecnologia e nanociência foi discutida por Richard P. Feynman, no ano de 1959. A nanociência começou a ser profundamente estudada a partir dos anos 1980, pelo engenheiro americano Kim Eric Drexler. É um campo de conhecimento transdisciplinar que está sendo desenvolvido e aplicado em diversas áreas, dentre elas, cabe ressaltar a área da saúde, principalmente no que tange à terapêutica e ao diagnóstico, já que é notória a necessidade de sistemas terapêuticos e de diagnósticos mais eficientes, sobretudo quanto à relação risco/benefício para os pacientes (Tobler, et al., 2020). A aplicação das nanopartículas no panorama biotecnológico e industrial têm impulsionado interesse na nanomedicina no âmbito de desenvolvimento de medicamentos mais seguros, eficazes e precisos, fornecendo benefícios substâncias e clinicamente significantes (Menck, et al., 2020). Alencar (2017) aponta que segundo a International Standards Organization (ISO), contempla pelo menos um dos seguintes aspectos na sua caracterização: o (i) entendimento e controle da matéria e processos em nanoescala, tipicamente, mas não exclusivamente, abaixo de 100 nanômetros em uma ou mais dimensões, em que o aparecimento de fenômenos dependentes de tamanho permite novas aplicações; e a (ii) utilização das propriedades dos materiais em nanoescala que são diferentes das propriedades dos átomos individuais, moléculas, ou dos materiais macroscópicos, criando materiais, dispositivos e sistemas melhores que exploram essas novas propriedades.

A inovação no setor farmacêutico pode surgir a partir da modificação de uma ou mais propriedades do composto, incluindo a estrutura química ou o método de síntese do princípio ativo, a forma farmacêutica, características farmacodinâmicas ou farmacocinéticas, bem como outras propriedades terapêuticas (Giuriatti, 2018). Svirkis (2013) traz as ideias de Figeiredo (2013) acerca da inovação na área de sistemas de liberação de fármacos (SLF) que estão surgindo a um ritmo muito mais rápido em relação as duas últimas décadas. Sbalqueiro (2018) mostra Fahning (2018) onde debate que tais dispositivos nanoestruturados visam disseminar substâncias com capacidade terapêutica e aumentar a eficácia dos mesmos no organismo através de sua liberação controlada e direcionamento celular, oferecendo inúmeras vantagens quando contrapostos com as formas farmacêuticas convencionais.

Os investimentos em nanociência e nanotecnologia estão presentes em todas as áreas do conhecimento e representam um investimento da ordem de bilhões de dólares, por parte dos órgãos e agências de fomento em pesquisa e desenvolvimento em todo o mundo (Moreira, 2013). Giuriatti (2018) apresentou que para Statnano (2018), na área farmacêutica, são 387 
produtos com nanotecnologia relacionados a medicamentos para humanos aplicados em todo mundo, com 75 empresas utilizando nanotecnologia em 19 países.

No Brasil, estudos relacionados à nanotecnologia vêm sendo incentivados pelo Conselho Nacional de Desenvolvimento Científico e Tecnológico (CNPq) e Ministério de Ciência e Tecnologia (MCT), desde 2001, quando foram criadas 4 redes de pesquisa em Nanociência e Nanotecnologia (Sant'anna, et al., 2013). O Brasil possui pesquisa na área, porém se comparar com o número de patentes publicadas, percebe-se que o domínio estrangeiro é indiscutível, pois é encabeçado por grandes multinacionais dos setores farmacêutico e cosmético que investem pesado em P\&D (Fialho, et al., 2016).

O objetivo deste revisar e investigar estudos sobre o uso da nanotecnologia em fármacos bem como o atual mapa Brasileiro e consequente explanação sobre a importância e contribuição de mecanismos como esses para o tratamento de doenças suas vantagens e desvantagens.

\section{Metodologia}

Trata-se de uma revisão da literatura disponível, na modalidade narrativa, com a abordagem qualitativa. Que Segundo Rother (2007), é uma categoria de artigos que têm um papel fundamental para a educação continuada pois, permitem ao leitor adquirir e atualizar o conhecimento sobre uma temática específica em curto espaço de tempo.

A coleta de dados foi realizada de forma qualitativa. Para Dias (2000), os métodos qualitativos são menos estruturados, lidam com informações mais subjetivas, amplas e com maior riqueza de detalhes do que os métodos quantitativos, a pesquisa qualitativa proporciona um conhecimento mais profundo e subjetivo.

Para a elaboração do estudo a busca por artigos sobre a temática foi realizada através das bases de dados, Scientific Electronic Library Online (SCIELO), Biblioteca Virtual em Saúde (BVS) e Google acadêmico. A busca foi realizada no período de junho a agosto de 2021. Os descritores em saúde utilizados conforme o DeCS foram: "Nanotecnologia", "Medicamentos e Tecnologia", "Nanoestruturas", "Nanotecnologia no Brasil" e "Nanotecnologia em Fármacos".

Como critérios de inclusão os artigos utilizados para acrescentar a explanação sobre o tema foram os que apresentassem abordagens sobre o uso da nanotecnologia em fármacos e aplicação no Brasil, publicados em português, Espanhol ou inglês, possuindo texto completo, disponível, gratuito e ter sido realizados nos últimos 10 anos, ou seja, publicados no período de 2011 a 2021. Como critério de exclusão não foram selecionados os artigos que fugissem do tema proposto bem como os que se repetiam nas bases de dados e não estivesse dentro do período proposto. Inicialmente foi realizada a leitura dos títulos e resumos de cada artigo, em seguida os artigos que se enquadraram no objetivo do estudo foram lidos na integra e explorados para estabelecimento de categorias e embasamento do estudo, tal sequência até a conclusão do trabalho está representado no fluxograma abaixo: 
Figura 1 - Fluxograma da seguência do trabalho.

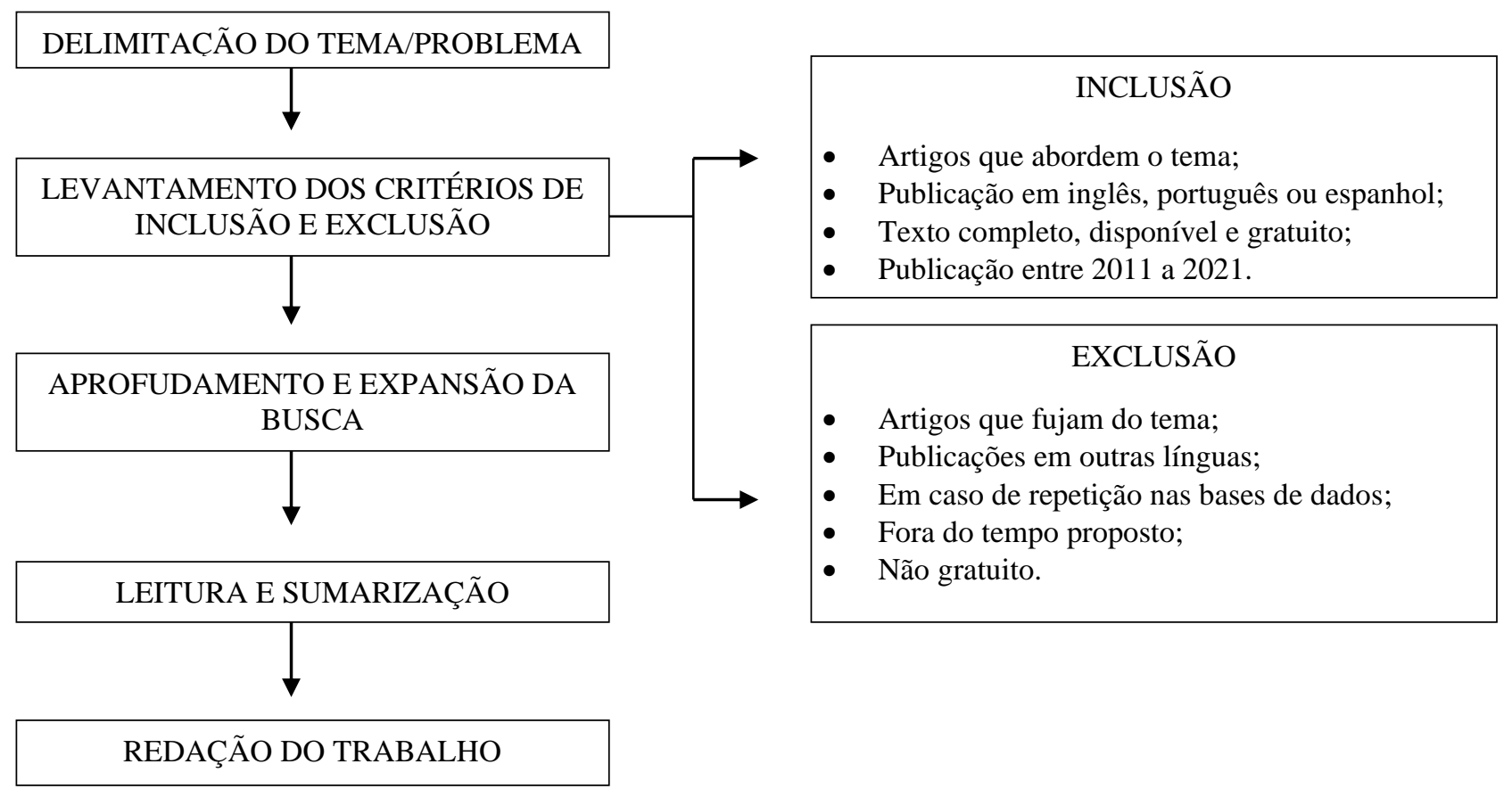

Fonte: Autores.

A presente revisão narrativa, qualitativa integra as perspectivas éticas, assegurando e tendo o compromisso de garantir a real autoria dos artigos nos quais serão utilizados como base de dados, empregando citações e referências de seus respectivos autores seguindo as normas da APA - American Psychological Association.

\section{Resultados}

Após toda a pesquisa utilizando os critérios de inclusão e exclusão já mencionados na metodologia, foi realizada uma seleção dos artigos a serem usados na explanação da temática objetivada e com a breve leitura dos títulos e resumos dos que restaram, pode-se excluir os artigos que fugiam do tema, chegando assim a um total de 25 publicações que deram o embasamento para o estudo desejado. Por fim para melhor compreensão dos resultados encontrados na literatura disponível foi feito um banco de dados com os resultados de cada estudo como apresentado na tabela 1 que contem o ano de publicação, os autores do trabalho, o título e as contribuições para o estudo. 
Tabela 1. Resultados Obtidos.

$\begin{array}{ccc}\text { ANO DE } & \text { AUTORES } & \text { TÍTULO } \\ \text { PUBLICAÇÃO } & & \text { CONTRIBUIÇÕES }\end{array}$

\begin{tabular}{|c|c|c|c|c|}
\hline 2011 & $\begin{array}{l}\text { Alice, L., } \\
\text { Ruppenthal, J. E., \& } \\
\text { Beck, R. C. R. }\end{array}$ & $\begin{array}{l}\text { A inovação } \\
\text { nanotecnologia } \\
\text { farmacêutica no } \\
\text { econômico do Bric. }\end{array}$ & $\begin{array}{r}\text { em } \\
\text { bloco }\end{array}$ & $\begin{array}{l}\text { Os autores apresentaram que o Brasil mesmo estando em uma posição } \\
\text { boa no ranking quando comparada a América Latina ainda assim } \\
\text { encontra-se estagnado no desenvolvimento de nanotecnologias } \\
\text { farmacêuticas, o país possuí investimento, porém não há o } \\
\text { desenvolvimento em si e que se precisa da criação de oportunidades } \\
\text { para o mercado dos produtos inovadores. }\end{array}$ \\
\hline
\end{tabular}

\begin{tabular}{|c|c|c|c|}
\hline 2017 & $\begin{array}{l}\text { Alencar, M. S. D. } \\
\text { M., Bochner, R., } \\
\text { Dias, M. F. F., \& } \\
\text { Antunes, A. M. D. } \\
\text { S. }\end{array}$ & $\begin{array}{l}\text { Análise da produção } \\
\text { científica brasileira sobre } \\
\text { nanotecnologia e saúde. }\end{array}$ & $\begin{array}{l}\text { O estudo traz que o cenário brasileiro aponta as universidades públicas } \\
\text { sendo as que mais publicam trabalhos na área da nanotecnologia e que } \\
\text { as parcerias internacionais há um maior destaque para a Fundação } \\
\text { Oswaldo Cruz e a Universidade Federal do Ceará. }\end{array}$ \\
\hline 2014 & $\begin{array}{l}\text { Batista, A. D. J. S., } \\
\text { \& Pepe, V. L. E. }\end{array}$ & $\begin{array}{l}\text { Os desafios da } \\
\text { nanotecnologia para a } \\
\text { vigilância sanitária de } \\
\text { medicamentos. }\end{array}$ & $\begin{array}{l}\text { O trabalho mostrou que a regulamentação dos medicamentos } \\
\text { nanotecnológicos terão desafios maiores e precisaram possuir testes por } \\
\text { pessoas especializadas, equipamentos sofisticados e a avaliação da } \\
\text { qualidade deverá abranger novos parâmetros como o tamanho da } \\
\text { partícula. }\end{array}$ \\
\hline
\end{tabular}

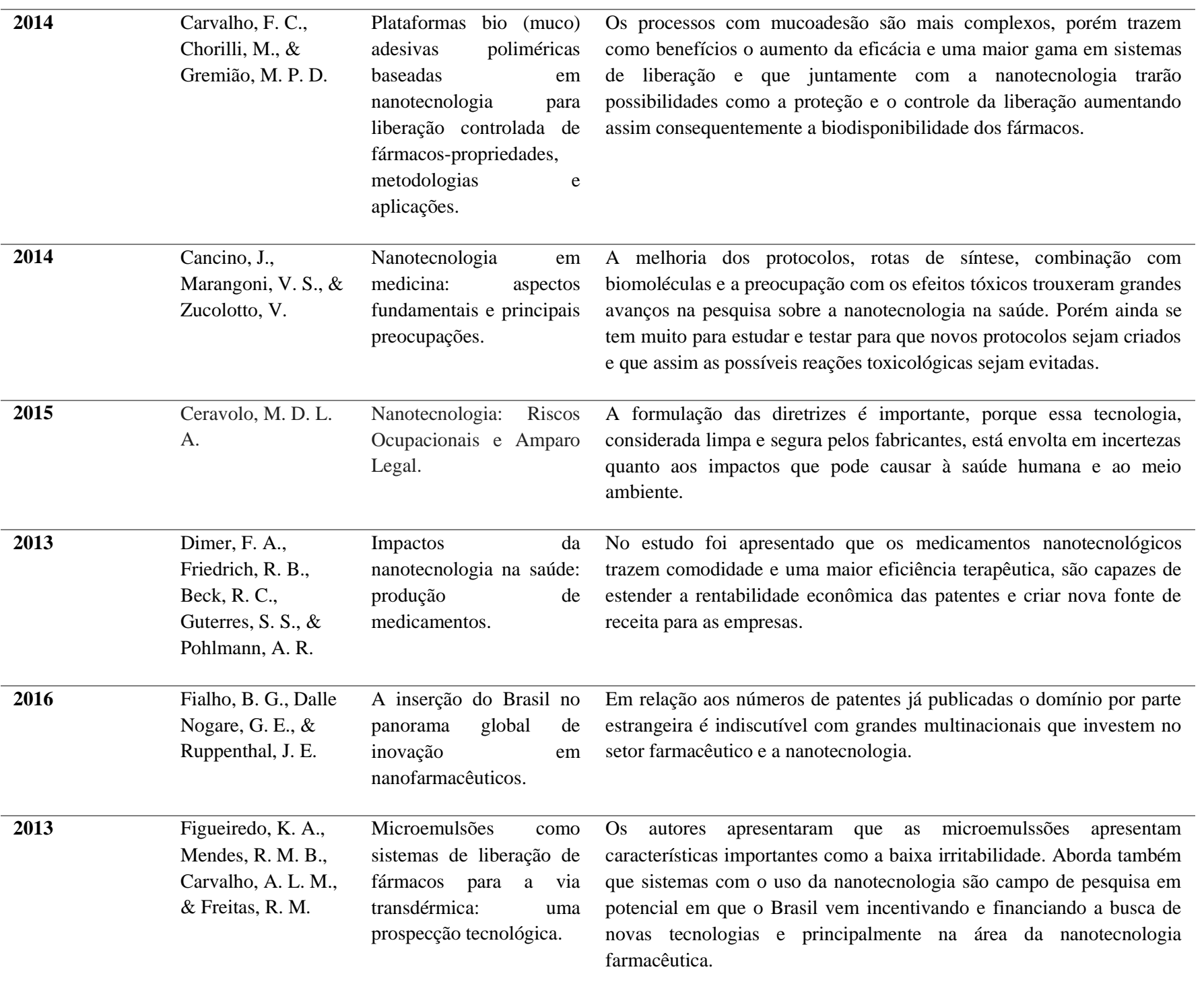


FREITAS, D. C., \& Aplicações

MUNIZ, B. V. nanotecnologia

fármacos para

tratamento do câncer de

mama.
O estudo trouxe diversas vantagens dos nanomedicamentos e que os

em principais fármacos utilizados no tratamento do câncer de mama em sua forma nanoestruturadas evidenciam esses benefícios como a biocompatibilidade, biodegradabilidade e seletividade, além de terem menores efeitos colaterais durante o tratamento.

Nanotecnologia em
fármacos: prospecção tecnológica no âmbito nacional e internacional.
Os Estados Unidos é o principal responsável pelas pesquisas em nanotecnologia, possuí alto investimento que se necessita em laboratórios, microscópios avançados para as análises das matérias em escala nanométrica. Porém os números de pesquisas são maiores que patentes o que significa dizer que os estudos não saem muito do papel.

\begin{tabular}{|c|c|c|c|}
\hline 2018 & Giuriatti, T. & $\begin{array}{lrr}\text { Mapa tecnológico } & \text { da } \\
\text { nanotecnologia } & \text { no } & \text { setor } \\
\text { brasileiro } & & \text { de } \\
\text { medicamentos } & & \text { em } \\
\text { humanos. } & & \end{array}$ & $\begin{array}{l}\text { Observou-se que a maior contribuição para o mercado brasileiro está } \\
\text { diretamente relacionada às parcerias firmadas entre ICTs e empresas. }\end{array}$ \\
\hline 2020 & $\begin{array}{l}\text { Giuriatti, T., De Prá, } \\
\text { M. A. A., Richartz, } \\
\text { F., \& Moreira, E. L. } \\
\text { G. }\end{array}$ & $\begin{array}{l}\text { Análise do } \text { P\&D } \\
\text { Brasileiro com Uso da } \\
\text { Nanotecnologia } \\
\text { Medicamentos para uso } \\
\text { humano: pesquisas, } \\
\text { tecnologias e produtos. }\end{array}$ & $\begin{array}{l}\text { Existe uma maior tendência ascendente de linhas de pesquisas voltadas } \\
\text { ao desenvolvimento de agentes antineoplásicos e imunomoduladores e } \\
\text { que a FIOCRUZ, a UNESP e a UFPE apresentaram um número } \\
\text { significativo de linhas de pesquisas em andamento. Devido a } \\
\text { necessidade de estudos pré-clínicos e clínicos o processo de } \\
\text { transformação da pesquisa em produtos é naturalmente mais lenta. }\end{array}$ \\
\hline 2014 & $\begin{array}{l}\text { Hermes, E. G. C., \& } \\
\text { de Oliveira Basto, P. } \\
\text { R. H. }\end{array}$ & $\begin{array}{l}\text { Nanotecnologia: } \\
\text { progresso científico, } \\
\text { material, global e } \\
\text { ético. Persona y Bioética. }\end{array}$ & $\begin{array}{l}\text { São poucos os estudos que defina as concentraçães seguras da } \\
\text { exposição aos nanomaterias e assim a elaboração das normas para } \\
\text { utilização e as regras para eliminação dos resíduos gerados. }\end{array}$ \\
\hline 2019 & $\begin{array}{l}\text { Hupffer, H. M., \& } \\
\text { Lazzaretti, L. L. }\end{array}$ & $\begin{array}{l}\text { Nanotecnologia e sua } \\
\text { regulamentação } \\
\text { Brasil. Revista Gestão e } \\
\text { Desenvolvimento. }\end{array}$ & $\begin{array}{l}\text { Os aspectos físico-químicos dos nanomateriais, são fundamentais, } \\
\text { visto que são essas propriedades que permitem novas funcionalidades } \\
\text { aos materiais já conhecidos pelos cientistas. A regulamentação } \\
\text { brasileira está focada em arrecadar fundos de investimento para } \\
\text { desenvolvimento da pesquisa e da indústria técnica nessa área e que é } \\
\text { um dos maiores desafios a serem debatidos entre o governo e os } \\
\text { diversos grupos de interesses brasileiros, considerando as } \\
\text { particularidades das características físico-química dos nanomateriais, } \\
\text { seus potenciais riscos e seu interesse comercial. }\end{array}$ \\
\hline
\end{tabular}
científica.

O estudo reforça a necessidade de avaliação da segurança e de formulação de regulamentações para o tema e criação de um órgão de regulamentação na área de nanotecnologia, propiciando a criação de uma legislação eficaz, pautada nos direitos constitucionais, em um meio ambiente ecologicamente equilibrado e na qualidade de vida e análise de impactos como também de riscos.

\begin{tabular}{|c|c|c|}
\hline 2020 & $\begin{array}{l}\text { Menck, C. F. M., } \\
\text { Wunderlich, G., } \\
\text { Romualdo, G. F., \& } \\
\text { Fotoran, W. L. }\end{array}$ & $\begin{array}{l}\text { Nanotecnologia aplicada } \\
\text { a entrega de fármacos } \\
\text { para superação de } \\
\text { obstáculos clínicos contra } \\
\text { tumores. Nanotecnologia } \\
\text { aplicada a entrega de } \\
\text { fármacos para superação } \\
\text { de obstáculos clínicos } \\
\text { contra tumores. }\end{array}$ \\
\hline
\end{tabular}

A aplicação das nanopartículas no panorama biotecnológico e industrial têm impulsionado interesse na nanomedicina no âmbito de desenvolvimento de medicamentos mais seguros, eficazes e precisos, fornecendo benefícios substâncias e clinicamente significantes. tratamentos terapêuticos, direciona o fármaco para ação no tumor evitando danos nas células saudáveis. 


\begin{tabular}{|c|c|c|c|}
\hline 2017 & Nolasco, L. G. & $\begin{array}{l}\text { Impactos } \\
\text { nanotecnologia na saúde } \\
\text { humana e no meio } \\
\text { ambiente. }\end{array}$ & $\begin{array}{l}\text { O trabalho traz pontos importantes a serem pensados em relação ao uso } \\
\text { das nanotecnologias como as necessidades vitais que são alteradas de } \\
\text { acordo com descobrimentos científicos e suas aplicações por exemplo, } \\
\text { o estudo de novas formulações químicas alucinógenas e o consequente } \\
\text { trafico de drogas. }\end{array}$ \\
\hline 2016 & $\begin{array}{l}\text { Rojas-Aguirre, Y., } \\
\text { Aguado-Castrejón, } \\
\text { K., \& González- } \\
\text { Méndez, I. }\end{array}$ & $\begin{array}{l}\text { La nanomedicina y los } \\
\text { sistemas de liberación de } \\
\text { fármacos: ¿ la } \quad \text { la } \\
\text { evolución de la terapia } \\
\text { contra el cáncer?. }\end{array}$ & $\begin{array}{l}\text { Apresenta um grande número de nanoplataformas que diagnosticam, } \\
\text { reconhecem um alvo terapêutico e fornecem uma ou mais drogas de } \\
\text { forma controlada. Devido ao conhecimento adquirido na área precisa-se } \\
\text { definir se com a nanomedicina esperamos uma revolução ou uma } \\
\text { evolução das terapias e como ela impactará na saúde da população. }\end{array}$ \\
\hline 2016 & $\begin{array}{l}\text { REIS, J. M. C. D., } \\
\text { PINHEIRO, M. F., } \\
\text { OTI, A. T., } \\
\text { FEITOSA-JUNIOR, } \\
\text { D. J. S., PANTOJA, } \\
\text { M. D. S., \& } \\
\text { BARROS, R. S. M. }\end{array}$ & $\begin{array}{l}\text { Informação tecnológica } \\
\text { relacionada aos pré e } \\
\text { probióticos com base no } \\
\text { registro das patentes: o } \\
\text { que há de novo? }\end{array}$ & $\begin{array}{l}\text { Apesar de muitos estudos sobre os probióticos e nanotecnologia serem } \\
\text { atraentes e mostrar que como alimentos funcionais, tem cada vez mais } \\
\text { pretendido diminuir os riscos e controlar a progressão de agravos à } \\
\text { saúde, ainda é incerto sua recomendação. }\end{array}$ \\
\hline 2018 & $\begin{array}{l}\text { Sbalqueiro, G., } \\
\text { Balvedi, L., } \\
\text { Bettiato, R., \& } \\
\text { Ribas, J. }\end{array}$ & $\begin{array}{l}\text { Uso da nanotecnologia } \\
\text { para o desenvolvimento } \\
\text { de fármacos. }\end{array}$ & $\begin{array}{l}\text { Traz a nonotecnologia na indústria farmacêutica como uma nova opção } \\
\text { com caminhos inovadores para aplicação e eficácia terapêutica. } \\
\text { Mostrou também que já é possível encontrar tratamentos para diversas } \\
\text { doenças sendo desenvolvidos, como Alzheimer, AIDS e câncer. Suas } \\
\text { vantagens fazem com que muitos estudos sejam feitos. }\end{array}$ \\
\hline 2013 & $\begin{array}{l}\text { Sant'Anna, L. D. S., } \\
\text { Alencar, M. S. D. } \\
\text { M., \& Ferreira, A. } \\
\text { P. }\end{array}$ & $\begin{array}{l}\text { Patenteamento em } \\
\text { nanotecnologia no Brasil: } \\
\text { desenvolvimento, } \\
\text { potencialidades } \\
\text { reflexões para o meio } \\
\text { ambiente e a saúde } \\
\text { humana. }\end{array}$ & $\begin{array}{l}\text { Devido a convergência tecnológica na nanotecnologia não há } \\
\text { mecanismo regulatório genérico que pode responder aos anseios em } \\
\text { investigação. Falta ainda muitas discussões sobre aos riscos e } \\
\text { consequências negativas. }\end{array}$ \\
\hline 2020 & $\begin{array}{l}\text { Silva, T. D. P., \& } \\
\text { Pinto, G. S. }\end{array}$ & $\begin{array}{l}\text { Nanotecnologia e sua } \\
\text { influência na evolução da } \\
\text { medicina. }\end{array}$ & $\begin{array}{l}\text { A nanotecnologia tem papel fundamental em novas técnicas médicas e } \\
\text { no desenvolvimento de medicamentos, com suas propriedades e } \\
\text { características únicas, influência diretamente na melhoria de } \\
\text { diagnósticos, tratamentos, visando e preservando sempre o conforto de } \\
\text { pacientes e auxiliando profissionais da saúde a melhorares suas } \\
\text { técnicas. }\end{array}$ \\
\hline 2020 & $\begin{array}{l}\text { Tobler, J. P., \& } \\
\text { Rocha, H. V. A. }\end{array}$ & $\begin{array}{l}\text { Bases regulatórias para a } \\
\text { avaliação da segurança de } \\
\text { medicamentos à base de } \\
\text { nanotecnologia. }\end{array}$ & $\begin{array}{l}\text { Testes toxicológicos preconizados atualmente pelas agências } \\
\text { reguladoras dos EUA, da União Europeia e do Brasil, apesar de } \\
\text { estarem alinhados, não são específicos para a avaliação de } \\
\text { nanomedicamentos. Com a base de dados disponível não se pode } \\
\text { garantir que a segurança risco/benefício. }\end{array}$ \\
\hline
\end{tabular}

Fonte: Regis, Feito e Guedes.

\section{Discussão}

No que se refere aos resultados dos estudos analisados, o estudo de Freitas (2015), traz que os nanomateriais são obtidos de formas orgânicas ou inorgânicas, sendo através dessas classificadas. Dentre essas classificações estão os de interesse farmacêutico, chamados de "Drug Delivery Systems" - Sistema de Liberação de drogas ou Fármacos. Cada um dos nanomateriais de interesse farmacêutico tem características específicas que serão aplicadas para a obtenção de um fármaco nanoestruturado. Essas têm vantagens por protegerem o fármaco da degradação do organismo, aumentar a sua solubilidade e biodisponibilidade, diminuir efeitos tóxicos, além de apresentarem controle e especificidade da liberação do fármaco.

Os materiais utilizados na obtenção de formulações nanotecnológicas são selecionados de acordo com suas características de biodegradabilidade, biocompatibilidade, capacidade para funcionalização de superfície, conjugação, 
complexação e encapsulamento. Os principais nanocarreadores engenheirados aplicados na terapêutica são: os lipossomas, as nanopartículas lipídicas, as micelas e as nanopartículas poliméricas. Por sua vez, os nanocristais representam uma estratégia de nanomização de fármacos pouco solúveis em água, de forma a aumentar a biodisponibilidade e propiciar vias de administração injetáveis (Dimer, 2013).

\section{Desvantagens}

As indústrias farmacêuticas têm investido na pesquisa e no desenvolvimento de novos medicamentos nanotecnológicos, que possuem sistemas nanoestruturados de liberação de fármacos. Como resultante dessas novas possibilidades de liberação dos fármacos, tem-se como vantagens a redução dos efeitos adversos e da toxicidade do fármaco, proporcionando maior conforto ao paciente e consequentemente, maior adesão ao tratamento. Contudo, esses sistemas também podem apresentar algumas desvantagens, como: 1) toxicidade dos produtos ou dos metabólitos oriundos da sua biodegradação; 2) preço mais elevado, devido ao custo do processo tecnológico, que pode ser compensado pela redução das doses; e, 3) possibilidade de permear barreiras biológicas e atingir órgãos nobres como o cérebro. Os benefícios das N\&N têm sido estudados e bem caracterizados, mas seus riscos ainda permanecem uma questão em aberto18. Essa falta de informações sobre os riscos advindos desta nova tecnologia é um dificultador para a tomada das decisões governamentais e a regulação da nanotecnologia (Batista, 2014).

Apesar dos avanços da nanomedicina em sistemas diagnósticos e terapêuticos, o potencial efeito na saúde humana devido à exposição prolongada ainda não foi estabelecido. A limitada quantidade de informações acerca dos efeitos tóxicos desses nanomaterias faz com que a nanomedicina enfrente várias questões regulatórias em um processo complexo e dispendioso, mas necessário, independentemente do nanomaterial ou aplicação. Uma das principais preocupações diz respeito a como as nanopartículas conseguem penetrar em membranas células mais facilmente, quando comparado a diversas outras moléculas ou fármacos. Será que essa maior facilidade pode ser alguma indicação de efeito tóxico? Essas dúvidas são o combustível dos estudos em nanotoxicologia, isto é, responder o maior número de questões sobre como é a interação entre nanomateriais e sistemas biológicos, e quais são as limitações e mecanismos associados a essa exposição em curto, médio e longo prazo (Cancino, 2014).

A falta de informação acerca dos efeitos nocivos das inovações tecnológicas no meio ambiente e na saúde humana serve de argumento para medidas de prudência. A identificação dos riscos é incipiente e a legislação existente é insuficiente e não se adapta às peculiaridades da nanotecnologia. Assim, no contexto atual, não existem avaliações "obrigatórias", exames específicos para definir os riscos da nanotecnologia (Ceravolo, 2015).

\section{Vantagens}

Discutindo sobre a incorporação de nanotecnologia em Sistemas mucoadesivos Carvalho (2014), conclui em seu estudo que podem promover um direcionamento e uma retenção mais específica do sistema de liberação. É possível projetar sistemas capazes de superar barreiras e melhorar a eficácia de fármacos. Esta plataforma tecnológica combinada com a nanotecnologia oferece possibilidades como a proteção e o controle da liberação e, portanto, são excelentes estratégias para aumentar a biodisponibilidade de fármacos.

Freitas (2020) traz em seu estudo que de acordo com Bucki (2016) a utilização da nanotecnologia no tratamento do câncer surge como uma inovação aos métodos já utilizados, cirúrgico, radioterápico e medicamentoso sistêmico, sendo principalmente o quimioterápico, pois os mesmos podem apresentar uma alta toxicidade, baixa seletividade, indução de células cancerígenas resistentes e recidiva do câncer. As nanopartículas possibilitam controlar e melhorar uma propriedade de maior interesse, direcionando um fármaco até as células tumorais, evitando a ação desses em células saudáveis. O conhecimento das 
Research, Society and Development, v. 10, n. 15, e32101522294, 2021

(CC BY 4.0) | ISSN 2525-3409 | DOI: http://dx.doi.org/10.33448/rsd-v10i15.22294

características básicas desses sistemas é importante para os profissionais preceptores, assim como o conhecimento dos medicamentos já disponíveis no mercado. Para o tratamento do câncer de mama, alguns fármacos já são utilizados, entretanto ainda são muito caros, dificultando assim o tratamento. Porém com o desenvolvimento de novas moléculas e a vetorização em novas nanopartículas, o acesso ao usuário será mais fácil, melhorando assim sua qualidade de vida (Moreira, 2013).

A aplicação das nanopartículas no panorama biotecnológico e industrial têm impulsionado interesse na nanomedicina no âmbito de desenvolvimento de medicamentos mais seguros, eficazes e precisos, fornecendo benefícios substâncias e clinicamente significantes (Menck, 2020). A nanotecnologia tem papel fundamental em novas técnicas médicas e no desenvolvimento de medicamentos. A tecnologia emergente em questão consegue utilizar de suas propriedades e características únicas, de tal maneira que consegue influenciar na melhoria de diagnósticos, tratamentos, visando e preservando sempre o conforto de pacientes e auxiliando profissionais da saúde a melhorares suas técnicas (Silva, 2020). A utilização e desenvolvimento da nanotecnologia envolvida na indústria farmacêutica é uma nova opção tecnológica que apresenta caminhos inovadores para a ampliação da eficácia terapêutica de fármacos já consagrados clinicamente, trazendo hoje impactos reais para a comunidade científica e a sociedade (Sbalqueiro, 2018).

\section{Situação Brasileira ao que se refere a utilização da nanotecnologia em fármacos e de sua regularização}

Para que uma nação atinja um nível adequado de desenvolvimento numa determinada área, é fundamental que além do conhecimento científico, sejam criadas oportunidades para a chegada ao mercado de produtos inovadores. O Brasil tem avançado consistentemente no desenvolvimento de relevantes ações em ciência, tecnologia e inovação, com resultados concretos na produção científica, tecnológica e formação de recursos humanos em áreas consideradas estratégicas. Porém há de se fazer muito mais, pois ciência e tecnologia não são capazes, sozinhas, de resolver os problemas dos países emergentes, contudo são componentes críticos e fundamentais para o seu desenvolvimento (Alice, 2011).

A partir deste novo milênio, o mundo e o Brasil vêm considerando a nanotecnologia como área estratégica. No Brasil essa consideração é facilmente demonstrada através dos programas de governo, entre outros: a Política Industrial, Tecnológica e de Comércio Exterior (PITCE), a criação de redes e institutos de pesquisa do CNPq, a Política de Desenvolvimento Produtivo (PDP), e o Plano Brasil Maior (PBM). O cenário brasileiro mostra que as universidades públicas são as que mais publicam na área, destacando-se instituições no estado de São Paulo, como a USP e a Unicamp, líderes em publicações, evidenciando a atuação forte do estado nesse setor. No entanto, observa-se que, do ponto de vista de parcerias internacionais em publicações, se destacam a Fundação Oswaldo Cruz e a Universidade Federal do Ceará. Apesar do crescimento desses temas de pesquisa, é possível constatar que $70 \%$ das publicações não foram redigidas em coautoria com pesquisadores de outros países, o que indica ainda pouca parceria internacional (Alencar, 2017). O autor Fialho (2016) em seu estudo confirma também que o Brasil possui pesquisa na área, porém se comparar com o número de patentes publicadas, percebe-se que o domínio estrangeiro é indiscutível, pois é encabeçado por grandes multinacionais dos setores farmacêutico e cosmético que investem pesado em P\&D.

Em sua pesquisa Fialkoski (2020) discutiu sobre patenteamento e mostrou que em 2010, foram realizados 4 depósitos na área de nanotecnologia em fármacos no Brasil, em 2011, foi o ano com a maior quantidade de depósitos com nanotecnologia, reflexo da grande quantidade de depósitos em nanotecnologia no ano de 2011. Apesar da quantidade considerável em 2011, no ano seguinte não houve depósitos na área. Em 2013, foram realizados 4 depósitos, em 2014 esse índice dobrou para 8 depósitos, em 2015 houve apenas 1 depósito, em 2016 foram 3, 2017 e 2018 teve 4 depósitos em cada ano. Logo entre 2010 e 2018 foram realizados 39 depósitos de patentes com nanotecnologia na subclasse A61K no Brasil. No Brasil os maiores depositantes foram a UNICAMP com 8, a USP com 8 e a UFRGS com 4 depósitos. 
Já Giuriatti (2020), trazendo a mesma temática mostrou que com relação aos depósitos de patentes, com prioridade no Brasil, observa-se que apenas 3,77\% de todos os depósitos brasileiros ocorreram até o ano de 2004 . Entre o período de 2004 até 2012 houve um crescimento no percentual de depósitos, de origem brasileira, de cerca de 32\%. A partir de 2013, até o ano de 2017, os depósitos de patentes cresceram na ordem de 50,94\%. De modo notório, houve uma significativa evolução no número de depósitos de patentes de origem nacional. Destaca-se a elevada taxa percentual de depósitos oriundos de instituições brasileiras de pesquisas (81,1\% dos depósitos do Brasil). Entre as principais aplicações terapêu-ticas observadas, destacam-se os agentes antineoplásicos, anti-infecciosos, imunomoduladores e dermatológicos.

Giuriatti (2018), enfatizou em seu estudo a importância das linhas de pesquisas das ICTs para o desenvolvimento de tecnologias por empresas brasileiras. Tais linhas de pesquisas forneceram estudos que resultaram no incremento de tecnologias depositadas em novos ensaios clínicos e no desenvolvimento de parcerias que propiciaram novos produtos para as empresas brasileiras. Contudo, é salutar que o mesmo resulte em incremento de parcerias com empresas nacionais, com as pesquisas vinculadas às necessidades da indústria brasileira, proporcionando, assim, novos produtos. O Brasil ainda caminha a passos lentos, com relação ao desenvolvimento de pesquisas que geram tecnologias por empresas nacionais nessa temática.

Para Nolasco (2017), Novas metodologias devem incorporar critérios de tamanho, forma, área de superfície, área de atividade e estrutura, além de exigir a construção de novos instrumentos de detecção, monitoramento e a caracterização adequada de nanomateriais, bem como os processos de compreensão que acontecem na superfície da nanopartícula quando em contato com os sistemas vivos, a fim de entender os possíveis efeitos toxicológicos, e, por conseguinte, contemplar as especificidades de controle e gerenciamento dos riscos em toda a cadeia produtiva e ciclo de vida de produtos e serviços com nanotecnologia.

Em seu levantamento Hupffer (2019), verificou-se que a regulamentação da nanotecnologia é um dos maiores desafios a serem debatidos entre o governo e os diversos grupos de interesses brasileiros, considerando as particularidades das características físico-química dos nanomateriais, seus potenciais riscos e seu interesse comercial. Assim como ocorre a nível mundial, observou-se que, mesmo com o crescimento do comércio de nanoprodutos, a regulamentação desses produtos é praticamente inexistente, centrada mais na regulação de organismos privados. Dessa forma, no Brasil, a Portaria 245 do Ministério da Ciência, Tecnologia e Inovação (MCTI) de 2012 pode ser considerada a única regulamentação vigente até o presente momento.

São necessários mais estudos para definir concentrações seguras de exposição aos nanomateriais, estabelecer normas de utilização e instituir regras adequadas para a eliminação dos resíduos associados à atividade nanotecnológica. A nanotecnologia é considerada a tecnologia-chave do século XXI; visto isso, é preciso estar atentos às condições de possibilidade do conhecimento e do que esperar, no caso presente e futuro da (nano) medicina, para que se traduza em práticas clínicas eficazes e seguras para o progresso benéfico à condição humana (Hermes, 2014).

No que se diz a respeito das dificuldades encontradas por parte dos pesquisadores, em seu artigo Jesus (2012), destacou que as alternativas que foram indicadas com maior frequência pelos especialistas foram: i) Falta de incentivos para os pesquisadores procurarem parcerias e o mercado; ii) Insuficiência de financiamento do poder público ou privado; iii) Dificuldade de regulamentação e de sua interpretação; iv) Dificuldade de competir com as indústrias internacionais.

Para Rojas (2016), o conceito de nanomedicina está presente em centenas de publicações, livros e monografias. O conhecimento nesse campo vem se acumulando tão rapidamente que é preciso questionar o caminho que a nanomedicina tomou, o curso que seguirá e como ela impactará na saúde da população. Para isso, é essencial analisar se com a nanomedicina esperamos uma revolução ou uma evolução das terapias para tratar as condições que até agora permanecem um desafio para a medicina. 
No processo do surgimento de novas patentes Reis (2016), em seu apresentou que as patentes, no âmbito da propriedade intelectual, são importantes por duas razões. Primeiro, porque elas constituem-se na forma mais tangível de propriedade intelectual e desfrutam de forte proteção legal. Segundo, porque os bancos de dados de patentes são poderosas fontes de informações tecnológicas a serem utilizadas na inteligência competitiva. Portanto, cada vez mais se deve estimular o ambiente de graduação e de pósgraduação para exploração desta fonte de conhecimento6,7. No Brasil, ainda há necessidade de ampliar os investimentos e proteção das propriedades industriais para geração de novos produtos, pois a quantidade de patentes depositadas ainda é incipiente. Há necessidade de maior incentivo governamental no sentido de criar parcerias entre as empresas e centros de pesquisas. O desenrolar de patentes em nanotecnologias no Brasil é fato os processos nanotecnológicos emergem significativamente no país, potencializando o desenvolvimento com a consequente disponibilização de produtos gerados (Sant'anna, 2013).

\section{Considerações Finais}

Com base na análise dos estudos apresentados, conclui-se que a nanotecnologia vem avançando ao longo dos anos em diversas áreas e na saúde não é diferente, é notório a busca de novas terapias medicamentosas e com o emprego de tecnologias, que proporcionam novos olhares para doenças encaradas anteriormente em outras perspectivas, por exemplo, o câncer. É necessário muito estudo, investimentos e incentivo para que surjam mais pesquisadores nesta área e que principalmente a pesquisa saia do papel para prática efetivamente e futuramente para a população proporcionando-a assim uma melhor qualidade de vida.

No que se diz respeito sobre a atual situação Brasileira sobre a produção de produtos farmacêuticos nanoestruturados é que apesar de ser considerado o melhor país da América Latina, o Brasil não avança no que se diz respeito sobre efetivamente a produção destes medicamentos, há uma grande complicação no processo de patenteamento dos já existentes e futuros medicamentos nanotecnológicos e uma não estruturação de leis que possam incentivar tal produção, pelo contrário, por não se ter algo tangível e bem estabelecido os pesquisadores por muitas vezes preferem realizar parcerias com outros países do que trazer tal produção ao território brasileiro.

Com esta revisão literária sobre a temática pode-se observar que são muitos os benefícios que a nanotecnologia pode proporcionar e que falta uma maior e mais profunda investigação para entendimento sobre os efeitos tóxicos que poderão estar associados bem como os efeitos adversos, mas que possivelmente se estudados poderiam ser amenizados e/ou controlados. Sendo assim a nanotecnologia empregada ao uso em fármacos deve ser entendida como a melhoria para as terapias medicamentosas visando sempre o bem-estar da população.

Para a realização de trabalhos futuros sugere-se que seja feita a busca em outras bases de dados e/ou acervos de instituições que ganham destaque no que se diz a respeito sobre estudo e desenvolvimentos de novos fármacos que tragam a nanotecnologia como meio de melhoria na eficácia terapêutica. Também apontamos como sugestão estudos que mostrem o impacto causado pela pandemia do covid-19 em aspectos como produção, investimento, desenvolvimento e pesquisa na área.

\section{Referências}

Alice, L., Ruppenthal, J. E., \& Beck, R. C. R. (2011). A inovação em nanotecnologia farmacêutica no bloco econômico do Bric. Revista ciência e tecnologia. 14(24/25), 77-84. https://www.lume.ufrgs.br/handle/10183/196634

Alencar, M. S. D. M., Bochner, R., Dias, M. F. F., \& Antunes, A. M. D. S. (2017). Análise da produção científica brasileira sobre nanotecnologia e saúde. https://www.arca.fiocruz.br/bitstream/icict/19053/2/ve_ALENCAR_Maria_Simone_de_Menezes_etal_2017.pdf

Batista, A. D. J. S., \& Pepe, V. L. E. (2014). Os desafios da nanotecnologia para a vigilância sanitária de medicamentos. Ciência \& Saúde Coletiva, 19, 21052114. https://www.scielosp.org/article/csc/2014.v19n7/2105-2114/ 
Carvalho, F. C., Chorilli, M., \& Gremião, M. P. D. (2014). Plataformas bio (muco) adesivas poliméricas baseadas em nanotecnologia para liberação controlada de fármacos-propriedades, metodologias e aplicações. Polímeros, 24(2), 203-213. https://www.scielo.br/j/po/a/ZjTRZ9TqxjkVXWVWMTGtxjn/?lang=pt

Cancino, J., Marangoni, V. S., \& Zucolotto, V. (2014). Nanotecnologia em medicina: aspectos fundamentais e principais preocupações. Química Nova, 37, 521-526. https://www.scielo.br/j/qn/a/dcxWV4RTSSjxDK3SsbdR8rR/?lang=pt

Ceravolo, M. D. L. A. (2015). Nanotecnologia: Riscos Ocupacionais e Amparo Legal. Revista Laborativa, 4(2), 53-63. https://core.ac.uk/download/pdf/233142367.pdf

Dias, C. A. (2000). Grupo focal: técnica de coleta de dados em pesquisas qualitativas. Informação \& Sociedade, 10(2). https://www.brapci.inf.br/_repositorio/2010/12/pdf_2fbfd6231b_0013748.pdf

Dimer, F. A., Friedrich, R. B., Beck, R. C., Guterres, S. S., \& Pohlmann, A. R. (2013). Impactos da nanotecnologia na saúde: produção de medicamentos. Química Nova, 36, 1520-1526. https://www.scielo.br/j/qn/a/56df7PWHsLXXb8sJ9m7LBqj/?lang=pt

Fialho, B. G., Dalle Nogare, G. E., \& Ruppenthal, J. E. (2016). A inserção do Brasil no panorama global de inovação em nanofarmacêuticos. Revista geintecgestao inovacao e tecnologias, 6(3), 3257-3263. https://www.revistageintec.net/index.php/revista/article/view/503

Figueiredo, K. A., Mendes, R. M. B., Carvalho, A. L. M., \& Freitas, R. M. (2013). Microemulsões como sistemas de liberação de fármacos para a via transdérmica: uma prospecção tecnológica. Revista geintec-gestao inovacao e tecnologias, 3(4), 036-046. https://www.revistageintec.net/index.php/revista/article/view/186

Freitas, D. C., \& Muniz, B. V. (2020). Aplicações da nanotecnologia em fármacos para o tratamento do câncer de mama. http://fait.revista.inf.br/imagens_arquivos/arquivos_destaque/NZJVMNpT8IdXjUO_2020-9-1-19-47-49.pdf

Fialkoski, D. (2020). Nanotecnologia em fármacos: prospecção tecnológica no âmbito nacional e internacional http://tede.unicentro.br:8080/jspui/handle/jspui/1547\#preview-link0

Giuriatti, T. (2018). Mapa tecnológico da nanotecnologia no setor brasileiro de medicamentos em humanos https://repositorio.ufsc.br/handle/123456789/205500

Giuriatti, T., De Prá, M. A. A., Richartz, F., \& Moreira, E. L. G. (2020). Análise do P\&D Brasileiro com Uso da Nanotecnologia em Medicamentos para Uso Humano: pesquisas, tecnologias e produtos. Cadernos de Prospecção, 13(1), 225. https://periodicos.ufba.br/index.php/nit/article/view/31806

Hupffer, H. M., \& Lazzaretti, L. L. (2019). Nanotecnologia e sua regulamentação no Brasil. Revista Gestão e Desenvolvimento, 16(3), 153-177. https://periodicos.feevale.br/seer/index.php/revistagestaoedesenvolvimento/article/view/1792/2432

Hermes, E. G. C., \& de Oliveira Basto, P. R. H. (2014). Nanotecnologia: progresso científico, material, global e ético. Persona y Bioética, 18(2), 107-118. https://www.redalyc.org/pdf/832/83232594002.pdf

Jesus, K. R. E. de, \& de Assis, O. B. G. (2012). Cenário atual das nanotecnologias no Brasil: prospecção da percepção da comunidade científica. In Embrapa Meio Ambiente-Artigo em anais de congresso (ALICE). In: WORKSHOP DA REDE DE NANOTECNOLOGIA APLICADA AO AGRONEGÓCIO, 6., 2012, Fortaleza. Anais... São Carlos: Embrapa Instrumentação; Fortaleza: Embrapa Agroindústria Tropical, $2012 . \quad$ p. 504-506. https://www.alice.cnptia.embrapa.br/alice/bitstream/doc/944559/1/2012AA017.pdf

Menck, C. F. M., Wunderlich, G., Romualdo, G. F., \& Fotoran, W. L. (2020). Nanotecnologia aplicada a entrega de fármacos para superação de obstáculos clínicos contra tumores. Nanotecnologia aplicada a entrega de fármacos para superação de obstáculos clínicos contra tumores, 1-388. https://www.atenaeditora.com.br/post-artigo/30401

Moreira, J. R. L. (2013). A nanotecnologia na liberação controlada de fármacos no tratamento do câncer de mama. https://bdm.unb.br/bitstream/10483/7034/1/2013_JoseRanclenissonLopesMoreira.pdf

Nolasco, L. G. (2017). Impactos da nanotecnologia na saúde humana e no meio ambiente. Revista Jurídica Direito, Sociedade e Justiça, 4(1). https://periodicosonline.uems.br/index.php/RJDSJ/article/view/1854/1505

Rother, E. T. (2007). Revisión sistemática X Revisión narrativa. https://www.scielo.br/j/ape/a/z7zZ4Z4GwYV6FR7S9FHTByr/?lang=es\&format=pdf

Rojas-Aguirre, Y., Aguado-Castrejón, K., \& González-Méndez, I. (2016). La nanomedicina y los sistemas de liberación de fármacos:i, la (r) evolución de la terapia contra el câncer? Educación química, 27(4), 286-291. https://reader.elsevier.com/reader/sd/pii/S0187893X16300295?toke n=C6CEDE5E81 8859A1F7293B0FE7D765B7232462E7D43D76B839B7204C268BE6FE37DC0FF59CE02E6BC03F2FA0B3E8FF65\&originRegion=us-east-

1 \&originCreation $=20211020023714$

Reis, J. M. C. D., Pinheiro, M. F., Oti, A. T., Feitosa-Junior, D. J. S., Pantoja, M. D. S., \& Barros, R. S. M. (2016). Informação tecnológica relacionada aos pré e probióticos com base no registro das patentes: o que há de novo? ABCD. Arquivos Brasileiros de Cirurgia Digestiva (São Paulo), $29(4), 279-281$. https://www.scielo.br/j/abcd/a/8nsKpMKFFncrs95R9Przd6d/?lang=pt

Sant'Anna, L. D. S., Alencar, M. S. D. M., \& Ferreira, A. P. (2013). Patenteamento em nanotecnologia no Brasil: desenvolvimento, potencialidades e reflexões para o meio ambiente e a saúde humana. Química Nova, 36, 348-353. https://www.scielo.br/j/qn/a/GQrZcsw7ZSZQvxghsJdVRkQ/?lang=pt\&format=pdf

Sbalqueiro, G., Balvedi, L., Bettiato, R., \& Ribas, J. (2018). Uso da nanotecnologia para o desenvolvimento de fármacos. Revista Saúde e Desenvolvimento, 12(10), 242-252. https://www.revistasuninter.com/revistasaude/index.php/saudeDesenvolvimento/article/view/881

Silva, T. D. P., \& Pinto, G. S. (2020). Nanotecnologia e sua influência na evolução da medicina. Revista Interface Tecnológica, 17(2), 269-280. https://revista.fatectq.edu.br/index.php/interfacetecnologica/article/view/982

Souza Marcone de, G. P. (2016). Nanotecnologia e nanociência: aspectos gerais, aplicações e perspectivas no contexto do Brasil. Revista Eletrônica Perspectivas da Ciência e Tecnologia, 7(2), 1. 